\title{
SOME COUNTEREXAMPLES AND PROPERTIES ON GENERALIZATIONS OF LINDELÖF SPACES
}

\section{FILIPPO CAMMAROTO}

Dipartimento di Matematica Universita' di Catania

Viale A Doria, 6

95100 Catania, ITALY

\section{GRAZIA SANTORO}

Dipartimento di Matematica

Universita' di Messina

Salita Sperone, n 31

98166 Sant' Agata, Messina, ITALY

(Received June 19, 1992 and in revised form September 27, 1995)

\begin{abstract}
In this paper we give some significative counterexamples to prove that some well known generalizations of Lindelof property are proper Also we give some new results, we introduce and study the almost regular-Lindelof spaces as a generalization of the almost-Lindelof spaces and as a new and significative application of the quasi-regular open subsets of [1].
\end{abstract}

KEY WORDS AND PHRASES: Lindelof space, almost Lindelof, weakly Lindelof and nearly Lindelof, semiregular and almost-regular space, regular cover

1980 AMS SUBJECT CLASSIFICATION CODES: 54C10, 54C20

\section{INTRODUCTION}

In literature there are several generalizations of the notion of Lindelof space [2] and these are studied separately for different reasons and purposes In 1959 Frolik [3] introduced the notion of weaklyLindelof spaces that, afterward, was studied by several authors: Comfort, Hindman and Negrepointis [4] in 1969, Hager [5] in 1969, Ulmer [6] in 1972, Woods [7] in 1976, Bell, Ginsburg and Woods [8] in 1978 About this topic in 1982 Balasubramanian [9] introduced and studied the notion of nearly-Lindelof spaces that is between Lindelof and weakly-Lindelof spaces In 1984 Willard and Dissanayake [10] gave the notion of almost $k$-Lindelof space, that for $k=\aleph_{0}$ we call almost-Lindelof, and that is between the nearly-Lindelof and weakly-Lindelof spaces. To be complete, it is useful to recall some recent papers of Pareek [11] which are an almost survey of all main generalizations of Lindelof spaces

In this paper we fix our attention on the main generalizations of Lindelof spaces, i e weaklyLindelof, almost-Lindelof and nearly-Lindelof spaces Our purpose is to study the relations between them and some new properties but, mainly, to construct some significative counterexamples to prove that the studied generalizations are proper.

Moreover, the counterexample 3.11, proving that there exist weakly-Lindelof spaces not almostLindelof, guides us to introduce and study a new generalization of Lindelof spaces, i e the almost regular-Lindelöf spaces These almost regular-Lindelöf spaces are a new and significative application of quasi-regular open subset introduced by the first author and Lo Faro [1] in 1981

We conclude the paper proposing the study of two new and natural generalizations of the almost regular-Lindelof spaces, i e the weakly regular-Lindelof and the nearly regular-Lindelof spaces

In particular, this paper is composed of four parts In $\S 1$ we study the nearly-Lindelof spaces as a generalization of Lindelof spaces (while Balasubramanian has studied them as a generalization of nearly compact spaces), we give some properties and a counterexample of a nearly-Lindelof not Lindelof space In $\S 2$ we study the subspaces and subsets nearly-Lindelof relative In $\S 3$ we give some properties of 
weakly-Lindelof spaces and a counterexample of weakly-Lindelof not nearly-Lindelof space, moreover, we study the almost-Lindelof spaces that are between nearly-Lindelof and weakly-Lindelof spaces, we give the necessary counterexamples and properties In the last section we introduce the notions of almost regular-Lindelof, weakly regular-Lindelof and nearly regular-Lindelof spaces

We have that the following implications hold

$$
\begin{array}{ccc}
\text { Nearly-L } & \Rightarrow \text { Almost-L } & \Rightarrow \text { Weakly-L } \\
\Downarrow & \Downarrow & \Downarrow
\end{array}
$$

Nearly R - L $\Rightarrow$ Almost R -L $\Rightarrow$ Weakly R -L

\section{PRELIMINARIES}

Throughout the present paper $X$ and $Y$ always denote topological spaces, $x$ an element of $X$ and $\mathcal{U}_{x}$ the neighborhoods filter of $x$ in $X$ The interior and the closure of any subset $A$ of $X$ will be denoted by $\operatorname{int}(A)$ or $\AA$ and $\operatorname{cl}(A)$ or $\bar{A}$ respectively.

If $A \subseteq S \subseteq X$, then $\operatorname{int}_{S}(A)$ and $\operatorname{cl}_{S}(A)$ will be used to denote respectively the interior and closure of $A$ in the subspace $S$ With $\left\{a_{\imath}\right\}_{\imath \geq \alpha}$ and $\left\{a_{\imath}\right\}_{\imath \in \mathbf{N}}$ we denote the set of all elements $a_{\imath}$ for each $i \geq \alpha$ and for each $i \in \mathbb{N}$ respectively

Recall some definitions $A=\bar{\AA}$ )

DEFINITION 1. A subset $A \subseteq X$ is called regularly open (resp. regularly closed) if $A=\stackrel{\circ}{A}$ (resp

The topology generated by the regularly open subsets of the space $(X, \tau)$ is denoted by $\tau^{*}$ and it is called semiregularization of $X$, if $\tau \equiv \tau^{*}$ then $X$ is said to be semiregular [12].

DEFINITION 2 [13]. A topological space $X$ is said to be almost regular if for each $x \in X$ and each regularly open neighborhood $U_{x} \in \mathcal{U}_{x}$, there exists a neighborhood $V_{x} \in \mathcal{U}_{x}$ such that $V_{x} \subset \bar{V}_{x} \subset U_{x}$, or, equivalently, if for any regularly closed set $C$ and any singleton $\{x\}$ disjoint from $C$, there exist disjoint open sets $U$ and $V$ such that $C \subseteq U$ and $x \in V$.

It is true that a space $X$ is regular if and only if it is semiregular and almost regular [13]

DEFINITION 3 [14]. A topological space $X$ is said to be nearly compact if every open cover $\left\{U_{\lambda}\right\}_{\lambda \in \Lambda}$ of $X$ admits a finite subfamily such that $X=\bigcup_{\imath=1}^{n} \frac{\circ}{U_{\lambda_{\imath}}}$.

DEFINITION 4 [2] Let $X$ be a topological space. A cover $\mathcal{V}=\left\{V_{j}\right\}_{j \in J}$ of $X$ is a refinement of another cover $\mathcal{U}=\left\{U_{\lambda}\right\}_{\lambda \in \Lambda}$ if for each $j \in J$ there exists an $\lambda(j) \in \Lambda$ such that $V_{j} \subset U_{\lambda(\jmath)}$.

DEFINITION 5 [2]. A family $\left\{U_{\lambda}\right\}_{\lambda \in \Lambda}$ of subsets of a topological space $X$ is locally finite if for every point $x \in X$ there exists a neighborhood $U_{x} \in \mathcal{U}_{x}$ such that the set $\left\{\lambda \in \Lambda: U_{x} \cap U_{\lambda} \neq \emptyset\right\}$ is finite

\section{§1. NEARLY LINDELÖF-SPACES}

DEFINITION 1.1 [9]. A topological space $X$ is said to be nearly-Lindelöf if for every open cover $\left\{U_{\lambda}\right\}_{\lambda \in \Lambda}$ of $X$ there exists a countable subset $\left\{\lambda_{n}\right\}_{n \in \mathbf{N}}$ of $\Lambda$ such that $X=\underset{n \in \mathbf{N}}{\cup} \overline{U_{\lambda_{n}}}$ (i e if every cover of $X$ by regularly open sets admits a countable subcover).

It is clear that every compact space is nearly-Lindelof, but the converse is not true (for example the real line $\mathbb{R}$ is nearly-Lindelof but it is not nearly compact).

Moreover, every Lindelöf space is nearly-Lindelöf but the converse is not true as the following example shows.

EXAMPLE 1.2. Let $\Omega$ be the smallest uncountable ordinal number and $A=[0, \Omega)$. The set $A$ has the property that for each $\alpha \in A$ the set $[0, \alpha)$ is countable, while $A$ is not. Let $X=\left\{a_{\imath \jmath}, c_{\imath}, a\right\}$ where $i \in A$ and $j \in \mathbb{N}$. We define in $X$ a topology such that the points $\left\{a_{\imath \jmath}\right\}$ are isolated and the fundamental system of neighborhoods of the points $\left\{c_{2}\right\}$ and $\{a\}$ are

$$
B_{c_{i}}^{n}=\left\{c_{\imath}, a_{\imath \jmath}\right\}_{\jmath \geq n} \text { and } B_{a}^{\alpha}=\left\{a, a_{\imath \jmath}\right\}_{\imath \geq \alpha, \jmath \in \mathbb{N}}
$$


respectively $X$ so topologized is Hausdorff but not Lindelof, in fact the open cover $\left\{B_{a}^{1}\right\} \cup\left\{B_{c_{a}}^{0}\right\}_{\text {, } A}$ has not countable subcover On the other hand, $X$ is nearly-Lindelof In fact, let $\left\{U_{\lambda}\right\}_{\lambda<\Lambda}$ be a cover of $X$ and $\bar{\lambda}$ such that $a \in U_{\lambda}$ Then $\left(X \backslash \frac{\dot{U_{\lambda}}}{\lambda}\right)$ is a countable set It follows that $X$ is nearly-Lindelof

PROPERTY 1.3. A space $(X, \tau)$ is nearly-Lindelof if and only if $\left(X, \tau^{*}\right)$ is Lindelof

COROLLARY 1.4. A nearly-Lindelof space $(X, \tau)$ is Lindelof if and only if it is semiregular

This is an improvement of [prop $5, \mathrm{~g}$ ] that holds only for regular spaces

PROPOSITION 1.5 [9] A topological space $X$ is nearly-Lindelof if and only if for any family $\left\{C_{\lambda}\right\}_{\lambda \in \Lambda}$ by regularly closed sets of $X$ with countable intersection property, the intersection $\cap_{\lambda_{t} \Lambda} C_{\lambda}$ is non-empty

PROPOSITION 1.6. Let $X$ be an almost regular and nearly-Lindelof space Then for every disjoint regularly closed $C_{1}$ and $C_{2}$ there exist two open sets $U$ and $V$ such that $U \cap V=\emptyset$ and $C_{1} \subset U, C_{2} \subset V$

PROOF. Since $X$ is almost regular, for each $x \in C_{1}$ there exists an open neighborhood $U_{x}$ such that $\overline{U_{x}} \cap C_{2}=\emptyset$ We can suppose $U_{x}$ to be regularly open The family $\left\{U_{x}\right\}_{x \in C_{1}} \cup\left\{X \backslash C_{1}\right\}$ is a regularly open cover of $X$ and, since $X$ is nearly-Lindelof, there exists a countable set of points $x_{1}, x_{2}, \ldots, x_{n}, \ldots$ of $X$ such that $X=\left(\cup_{n \in \mathbb{N}}^{\cup} U_{x_{n}}\right) \cup\left(X \backslash C_{1}\right)$ It follows that for each $n \in \mathbb{N} C_{1} \subset \underset{n \in \mathbb{N}}{\cup} U_{x_{n}}$ and $\overline{U_{x_{n}}} \cap C_{2}=\emptyset$ Analogously there exists a family of regular open sets $\left\{V_{y_{n}}\right\}$ such that $C_{2} \subset \underset{n \in \mathbb{N}}{\cup} V_{y_{n}}$ and $\overline{V_{y_{n}}} \cap C_{1}=\emptyset \quad$ Let $G_{n}=U_{x_{n}} \backslash\left(\bigcup_{\imath=1}^{n} \overline{V_{y_{i}}}\right), H_{n}=V_{y_{n}} \backslash\left(\bigcup_{\imath=1}^{n} \overline{U_{x_{i}}}\right)$ and $U=\cup_{n \in \mathbb{N}} G_{n}, V=\cup_{n \in \mathbb{N}} H_{n} \quad U$ and $V$ so constructed are the open sets that we are looking for

DEFINITION 1.7 [15] A space $X$ is said to be nearly paracompact if every cover of $X$ by regularly open sets admits a locally finite refinement

PROPOSITION 1.8 Let $X$ be an almost regular and nearly-Lindelof space Then $X$ is nearly paracompact

PROOF Let $\left\{U_{\lambda}\right\}_{\lambda \in \Lambda}$ be a cover of $X$ by regularly open sets For each $x \in X$ and $\bar{\lambda} \in \Lambda$ such that $x \in U_{\bar{\lambda}}$ there exists an open neighborhood $U_{x}$ of $x$ such that $\overline{U_{x}} \subset U_{\bar{\lambda}}$ We can suppose that $U_{x}$ is regularly open so $\left\{U_{x}\right\}_{x \in X}$ is a regular open cover of $X$. Since $X$ is nearly-Lindelof, there exists a countable set of points $x_{1}, x_{2}, \ldots, x_{n}, \ldots$ of $X$ such that $X=\cup_{n \in \mathbb{N}} U_{x_{n}}$ For each $n \in \mathbb{N}$ choose a $\lambda_{n} \in \Lambda$ such that $\overline{U_{x_{n}}} \subset U_{\lambda_{n}}$ and put $V_{n}=U_{\lambda_{n}} \backslash\left(\bigcup_{\imath=1}^{n-1} \overline{U_{x_{i}}}\right)$. By construction $\left\{V_{n}\right\}_{n \in \mathbf{N}}$ is a refinement of $\left\{U_{\lambda}\right\}_{\lambda \in \Lambda}$ and it is a locally finite family. In fact, let $x \in X$. Then there exist $U_{x_{p}}$ (since $\left\{U_{x_{n}}\right\}_{n \in \mathbf{N}}$ is a cover of $X$ ) and $U_{\lambda_{p}}$ such that $x \in U_{x_{p}} \subset U_{\lambda_{p}}$. We will prove that $U_{x_{p}}$ intersects at most finitely many members of the family $\left\{V_{n}\right\}_{n \in \mathbf{N}}$ Since

$$
V_{1}=U_{\lambda_{1}}, V_{2}=U_{\lambda_{2}} \backslash \overline{U_{x_{1}}}, \ldots, V_{p+1}=U_{\lambda_{p+1}} \backslash\left\{\overline{U_{x_{1}}} \cup \ldots \cup \overline{U_{x_{p}}}\right\},
$$

then $U_{x_{p}}$ is not contained in $V_{r}$ for each $r \geq p+1$ while $U_{x_{p}} \subset V_{p}$. So $U_{x_{p}} \cap V_{r}=\emptyset$ for each $r \geq p+1$, therefore $U_{x_{p}}$ intersects at most a finite number of sets $V_{n}$

PROPOSITION 1.9. Let $X$ be a nearly-Lindelöf space and $Y$ a nearly compact space Then $X \times Y$ is nearly-Lindelof.

PROOF. Let $\left\{U_{\lambda}\right\}_{\lambda \in \Lambda}$ be a cover of $X \times Y$ by regularly open sets Without loss of generality, we can suppose $U_{\lambda}=V_{\lambda} \times W_{\lambda}$ where $V_{\lambda}$ and $W_{\lambda}$ are regularly open sets in $X$ and $Y$ respectively Fix $x \in X$, for each $y \in Y$ there exists $\lambda_{y} \in \Lambda$ such that $(x, y) \in V_{\lambda_{y}} \times W_{\lambda_{y}}$ The family $\left\{W_{\lambda_{y}}\right\}_{y \in Y}$ is a cover of $Y$ by regularly open sets and, since $Y$ is nearly compact, it admits a finite subcover Let $Y=W_{\lambda_{y_{1}}} \cup \ldots \cup W_{\lambda_{y_{n}}}$ Put $H_{x}=V_{\lambda_{y_{1}}} \cap \ldots \cap V_{\lambda_{y_{r}}}$ and $r(x)=\left\{\lambda_{y_{1}}, \ldots, \lambda_{y_{r}}\right\} \quad H_{x}$ is a regularly open set of $X$ and hence the family $\left\{H_{x}\right\}_{x \in X}$ is a regularly open cover of $X$ Since $X$ is nearly-Lindelof, there exists a countable set of points $x_{1}, x_{2}, \ldots, x_{n}, \ldots$ of $X$ such that $X=\cup_{n \in \mathbb{N}} H_{x_{n}}$, hence 


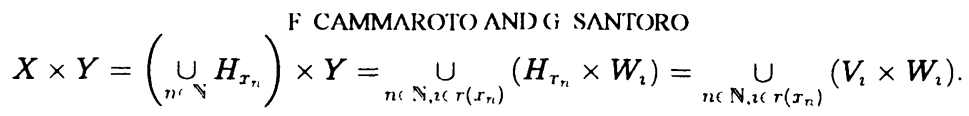

Since the last member is a countable family, we have that $X \times Y$ is nearly-Lindelof

REMARK 1.10 In general the product of two nearly-Lindelof spaces is not nearly-Lindelof In fact, let $K$ be the Sorgenfrey line $K$ is normal, and hence regular, and Lindelof and therefore it is nearly-Lindelof The product $K \times K$ is regular, but it is not Lindelof [2, 38 15] and therefore it cannot be nearly-Lindelof (see Corollary 14$)$

\section{§2. NEARLY-LINDELÖF SUBSPACES AND SUBSETS}

A subset $S$ of a space $X$ is said to be nearly-Lindelof if $S$ is nearly-Lindelof as subspace of $X$ (i e $S$ is nearly-Lindelof with respect to the inducted topology from the topology of $X$ )

DEFINITION 2.1 A subset $S$ of a space $X$ is said to be nearly-Lindelof relative to $X$ if for every cover $\left\{U_{\lambda}\right\}_{\lambda \in \Lambda}$ by open sets of $X$ such that $S \subset \underset{\lambda \in \Lambda}{\cup} U_{\lambda}$, there exists a countable subset $\left\{\lambda_{n}\right\}_{n \in N} \subset \Lambda$ such that $S \subset \underset{n \subset \mathbb{N}}{\cup} \frac{\circ}{U_{\lambda_{n}}}$

PROPOSITION 2.2 [9] Let $X$ be a space and $A$ an open subset of $X$ Then $A$ is nearly-Lindelof if and only if it is nearly-Lindelof relative to $X$

LEMMA 2.3 [9] Let $B$ be a regularly closed subset of a nearly-Lindelof space $X \quad$ Then $C$ is nearly-Lindelöf relative to $X$

COROLLARY 2.4 [9] A clopen of a nearly-Lindelof space is nearly-Lindelof

PROPERTY 2.5 Let $X$ be an extremally disconnected space (i e the closure of an open set is open [2]) and $S \subseteq X$ If $S$ is nearly-Lindelof then $S$ is nearly-Lindelöf relative to $X$

PROOF. Let $\left\{U_{\lambda}\right\}_{\lambda \in \Lambda}$ be an open family of $X$ such that $S \subset \underset{\lambda \in \Lambda}{\cup} U_{\lambda}$ Consider $V_{\lambda}=S \cap U_{\lambda}$ for each $\lambda \in \Lambda$, then $\left\{V_{\lambda}\right\}_{\lambda \in \Lambda}$ is an open cover of $S$. By hypothesis there exists a countable subfamily $\left\{V_{\lambda_{n}}\right\}_{n \in \mathbf{N}}$ such that $S=\bigcup_{n \in \mathbf{N}} \operatorname{int}_{S} \mathrm{cl}_{S}\left(V_{\lambda_{n}}\right)$. Since for each $n \in \mathbb{N} V_{\lambda_{n}} \subset U_{\lambda_{n}}$, then $\overline{V_{\lambda_{n}}} S \subset \overline{U_{\lambda_{n}}} X$ Since $X$ is extremally disconnected then int $\operatorname{cl}_{S}\left(V_{\lambda_{n}}\right) \subset \operatorname{int}_{X} \operatorname{cl}_{X}\left(U_{\lambda_{n}}\right)=\operatorname{cl}_{X}\left(U_{\lambda_{n}}\right) \quad$ This proves that $S \subseteq \underset{n \in \mathbb{N}}{\cup}$ $\frac{\circ}{U_{\lambda_{n}}}$, i.e. $S$ is nearly-Lindelöf relative to $X$.

REMARK 2.6. In general a closed subset of a nearly-Lindelof space is neither nearly-Lindelöf nor nearly-Lindelof relative to the space as the subset $\left\{c_{\imath}\right\}_{\imath \in A}$ in Example 1.2 shows

PROPOSITION 2.7. Let $X$ be a space and $S \subset X$. The following are equivalent

(i) $S$ is nearly-Lindelöf relative to $X$;

(ii) for every family by regularly open sets of $X$ that cover $S$, there exists a countable subfamily covering $S$;

(iii) for every family $\left\{C_{\lambda}\right\}_{\lambda \in \Lambda}$ by regularly closed sets of $X$ such that $\left(\underset{\lambda \in \Lambda}{\cap} C_{\lambda}\right) \cap S=\emptyset$, there exists a countable subset of indices $\left\{\lambda_{n}\right\}_{n \in \mathbf{N}} \subset \Lambda$ such that $\left(\underset{n \in \mathbf{N}}{\cap} C_{\lambda_{n}}\right) \cap S=\emptyset$

PROOF. (i) $\Rightarrow$ (ii) It is obvious by the definition.

(ii) $\Rightarrow$ (iii). Let $\left\{C_{\lambda}\right\}_{\lambda \in \Lambda}$ be a regularly closed family in $X$ such that $\left(\underset{\lambda \in \Lambda}{\cap} C_{\lambda}\right) \cap S=\emptyset$. Then $S \subset X \backslash\left(\underset{\lambda \in \Lambda}{\cap} C_{\lambda}\right)=\cup_{\lambda \in \Lambda}\left(X \backslash C_{\lambda}\right)$; hence $\left\{X \backslash C_{\lambda}\right\}_{\lambda \in \Lambda}$ is a regularly open family covering $S$, then there exists a countable subfamily $\left\{X \backslash C_{\lambda_{n}}\right\}_{n \in \mathbb{N}}$ such that $S \subset \underset{n \in \mathbf{N}}{\cup}\left(X \backslash C_{\lambda_{n}}\right)$, i e $\left(\underset{n \in \mathbb{N}}{\cap} C_{\lambda_{n}}\right) \cap S=\emptyset$

(iii) $\Rightarrow$ (i) Let $\left\{U_{\lambda}\right\}_{\lambda \in \Lambda}$ be a family by open subsets of $X$ such that $S \subset \underset{\lambda \in \Lambda}{\cup} U_{\lambda}$ Then $S \subset B 2 \underset{\lambda \in \Lambda}{\cup} U_{\lambda} \subset \underset{\lambda \in \Lambda}{\cup} \stackrel{\circ}{U_{\lambda}}$, therefore $\left(X \backslash\left(\cup_{\lambda \in \Lambda} \stackrel{\circ}{U_{\lambda}}\right)\right) \cap S=\emptyset$, i e $\underset{\lambda \in \Lambda}{\cap}\left(X \backslash \frac{\circ}{U_{\lambda}}\right) \cap S=\emptyset \quad$ By hypothesis there exists a countable subfamily $\left\{X \backslash \overline{U_{\lambda_{n}}}\right\}_{n \in \mathbf{N}}$ such that $\bigcap_{n \in \mathbf{N}}\left(X \backslash \overline{U_{\lambda_{n}}}\right) \cap S=\emptyset$ and therefore $\left(\mathrm{X} \backslash\left(\cup_{\mathrm{n} \in \mathbf{N}} \frac{\circ}{\mathrm{U}_{\lambda_{\mathrm{n}}}}\right)\right) \cap S=\emptyset$, i.e $S \subset \underset{n \in \mathbb{N}}{\cup} \frac{\circ}{\bar{U}_{\lambda_{n}}}$. This completes the proof 
PROPOSITION 2.8. A space $(X, \tau)$ is open hereditarily nearly-Lindelof if and only if any $A \in \tau^{*}$ is nearly-Lindelof

PROOF. Let $B \subset X$ be an open subset of $X$ By Proposition 22 it is sufficient to prove that $B$ is nearly-Lindelof relative to $X$ Let $\left\{U_{\lambda}\right\}_{\lambda \leftarrow A}$ be a family by regularly open sets of $X$ such that $B \subset \cup_{\lambda \leftarrow \Lambda} U_{\lambda}$ The set $A=\cup_{\lambda \in \Lambda} U_{\lambda}$ belongs to $\tau^{*}$, so by hypothesis $\mathrm{A}$ is nearly-Lindelof Hence there exists a countable subfamily $\left\{U_{\lambda_{n}}\right\}_{n \in \mathbb{N}}$ of $\left\{U_{\lambda}\right\}_{\lambda \in \Lambda}$ such that $A=\cup_{n \in \mathbb{N}} U_{\lambda_{n}}$ and therefore $B \subset \cup_{n \in \mathbb{N}} U_{\lambda_{r}}$ Conversely, let $X$ be open hereditarily nearly-Lindelof Since $\tau^{*} \subset \tau$, it is obvious that any $A \in \tau^{*}$ is nearly-Lindelof

THEOREM 2.9. Let $f: X \rightarrow Y$ be a closed continuous function and, for each $y \in Y$, let $f^{1}(y)$ be nearly-Lindelof relative to $X$ If $Y$ is nearly-Lindelof then $X$ is nearly-Lindelof

PROOF. Let $\left\{C_{\lambda}\right\}_{\lambda \in \Lambda}$ be a family of regularly closed subsets of $X$ with countable intersection property Let $M=\Lambda^{\mathrm{N}}$, i e each $\mu \in M$ is of the form $\mu=\left(\lambda_{1}, \lambda_{2}, \ldots, \lambda_{n}, \ldots\right)$ Put $C_{\mu}=\bigcap_{n \in \mathbb{N}} C_{\lambda_{n}} \neq \emptyset$ The family $\left\{C_{\mu}\right\}_{\mu \in M}$ is a family by closed subsets of $X$ with countable intersection property and also the family $\left\{f\left(C_{\mu}\right)\right\}_{\mu \in M}$ in $Y$ is so Since $Y$ is nearly-Lindelof, by Proposition 15 there exists $\bar{y} \in Y$ such that $\bar{y} \in f\left(C_{\mu}\right)$ for each $\mu \in M \quad$ It follows that $f^{-1}(\bar{y}) \cap C_{\mu} \neq \emptyset$ for each $\mu \in M$, hence $f^{-1}(\bar{y})$ intersects all countable intersections of $C_{\lambda}$ with $\lambda \in \Lambda$ Since $f^{-1}(\bar{y})$ is nearly-Lindelof relative to $X$, by Proposition 27 (iii), we have $\left(\underset{\lambda \in \Lambda}{\cap} C_{\lambda}\right) \cap f^{-1}(\bar{y}) \neq \emptyset$ and thus $\underset{\lambda \in \Lambda}{\cap} C_{\lambda} \neq \emptyset$ This, by Proposition 15 , implies that $X$ is nearly-Lindelof

REMARK 2.10. Recall that, for a topological space $X$, the Lindelof number $l(X)$ is defined as the smallest cardinal number $\lambda$ such that every open cover of $X$ admits a subcover of cardinality $\lambda$ It is natural to generalize this notion to nearly-Lindelof space defining the nearly-Lindelof number of $X \mathrm{nl}(X)$ to be the smallest cardinal number $\mu$ such that every regularly open cover of $X$ admits a subcover of cardinality $\mu$

Obviously $n l(X) \leq l(X)$ and this inequality can be proper For this purpose we can see Example 12

\section{§3. ALMOST-LINDELÖF AND WEAKLY-LINDELÖF SPACES}

DEFINITION 3.1 [10] A topological space $X$ is called almost-Lindelöf if every open cover $\left\{U_{\lambda}\right\}_{\lambda \in \Lambda}$ of $X$ admits a countable subfamily such that $X=\cup \overline{n \in \mathbb{N}} \overline{U_{\lambda_{n}}}$

DEFINITION 3.2 [3] A topological space $X$ is said to be weakly-Lindelof if for every open cover $\left\{U_{\lambda}\right\}_{\lambda \in \Lambda}$ of $X$ there exists a countable subfamily such that $X=\overline{\bigcup_{n \in \mathbb{N}} U_{\lambda_{n}}}$

PROPOSITION 3.3. A topological space $X$ is weakly-Lindelof if and only if for any family of closed subsets of $X\left\{C_{\lambda}\right\}_{\lambda \in \Lambda}$ such that $\bigcap_{\lambda \in \Lambda} C_{\lambda}=\emptyset$ there exists a countable subfamily $\left\{C_{\lambda_{n}}\right\}_{n \in \mathbb{N}}$ such that $\operatorname{int}\left(\underset{n \in \mathrm{N}}{\cap} C_{\lambda_{n}}\right)=\emptyset$.

PROOF. Let $\left\{C_{\lambda}\right\}_{\lambda \in \Lambda}$ be a family of closed subsets of $X$ such that $\cap C_{\lambda}=\emptyset$ Then $X=\cup_{\lambda \in \Lambda}\left(X \backslash C_{\lambda}\right)$, so by hypothesis there exists a countable subfamily such that $X={\underset{n \in \mathbb{N}}{\cup}\left(X \backslash C_{\lambda_{n}}\right)}_{\text {Hence }}$ $X \backslash \overline{n \in \mathbf{N}} \overline{\bigcup_{\lambda \in \Lambda}\left(X \backslash C_{\lambda_{n}}\right)}=\emptyset$, i e $\operatorname{int}\left(X \backslash\left(\cup_{n \in \mathbf{N}}\left(X \backslash C_{\lambda_{n}}\right)\right)\right)=\operatorname{int}\left(\cap_{n \in \mathbf{N}} C_{\lambda_{n}}\right)=\emptyset \quad$ Conversely, let $\left\{U_{\lambda}\right\}_{\lambda \in \Lambda}$ be an open cover of $X$ Then $\bigcap_{\lambda \in \Lambda}\left(X \backslash U_{\lambda}\right)=\emptyset$ and therefore there exists a countable subfamily such that $\operatorname{int}\left(\underset{n \in \mathbb{N}}{\cap}\left(X \backslash U_{\lambda_{n}}\right)\right)=\emptyset$. So

$$
X=X \backslash \operatorname{int}\left(\bigcap_{n \in \mathbb{N}}\left(X \backslash U_{\lambda_{n}}\right)\right)=\overline{X \backslash\left(\underset{n \in \mathbb{N}}{\cap}\left(X \backslash U_{\lambda_{n}}\right)\right)}=\overline{\cup_{n \in \mathbb{N}} U_{\lambda_{n}}}
$$

PROPOSITION 3.4. Let $X$ be a topological space For the following conditions

(i) $X$ is weakly-Lindelof, 
(ii) any cover $\left\{U_{\lambda}\right\}_{\lambda^{\prime} .1}$ of $X$ by regularly open sets of $X$ admits a countable subfamily with dense union in $X$,

(iii) if $\left\{C_{\lambda}\right\}_{\lambda<\Lambda}$ is a family of regularly closed subsets of $X$ such that $\underset{\lambda \subset \Lambda}{\cap} C_{\lambda}=\emptyset$, then there exists a countable subfamily such that int $\left(\cap \cap_{n} C_{\lambda_{n}}\right)=\emptyset$,

we have that (i) $\Rightarrow$ (ii) $\Rightarrow$ (iii) $\Rightarrow$ (ii) and if $X$ is semiregular then (ii) $\Rightarrow$ (i)

PROOF. (i) $\Rightarrow$ (ii) is obvious from the definition The proof of (ii) $\Leftrightarrow$ (iii) is quite similar to the proof of Proposition 33 replacing open cover with a regularly open cover of $X$ We will prove the implication (ii) $\Rightarrow$ (i) when $X$ is semiregular Let $\left\{U_{\lambda}\right\}_{\lambda \in \Lambda}$ be an open cover of $X$ By hypothesis we can suppose any $U_{\lambda}$ to be regularly open Then there exists a countable subfamily $\left\{U_{\lambda_{n}}\right\}_{n, \mathbb{N}}$ such that $\overline{\cup_{n \in \mathbb{N}} U_{\lambda_{r}}}=X$ This completes the proof

Obviously, if a space is nearly-Lindelof then it is almost-Lindelof and if a space is almost-Lindelof then it is weakly-Lindelof But the following example shows that weakly-Lindelof property or almostLindelof property does not imply the nearly-Lindelof property

EXAMPLE 3.5. Let $\Omega$ be the smallest uncountable ordinal number and $A=[0, \Omega)$ as in Example 12 Let $X=\left\{a_{\imath \jmath}, b_{\imath \jmath}, c_{\imath}, a, b\right\}$ where $i \in A$ and $\jmath \in \mathbb{N}$ Consider in $X$ the topology such that the points $\left\{a_{\imath \jmath}\right\}$ and $\left\{b_{\imath \jmath}\right\}$ are isolated and the fundamental system of neighborhoods of the points $\left\{c_{\imath}\right\},\{a\}$ and $\{b\}$ are

$$
B_{c_{\imath}}^{n}=\left\{c_{\imath}, a_{\imath \jmath}, b_{\imath \jmath}\right\}_{\jmath \geq n}, \quad B_{a}^{\alpha}=\left\{a, a_{\imath \jmath}\right\}_{\imath \geq \alpha, \jmath \in \mathbb{N}} \text { and } B_{b}^{\alpha}=\left\{b, b_{\imath \jmath}\right\}_{\imath \geq \alpha, \jmath \in \mathbf{N}}
$$

respectively $X$ so topologized is Hausdorff and semiregular but it is not nearly-Lindelof as we can see considering the regularly open cover $\left(\cup_{\imath \in A} B_{c_{1}}^{0}\right) \cup B_{a}^{1} \cup B_{b}^{1} \quad$ But $X$ is weakly-Lindelof Indeed, let $\left\{U_{\lambda}\right\}_{\lambda \in \Lambda}$ be an open cover of $X$ Then there exist $\lambda_{1}, \lambda_{2} \in \Lambda$ such that $a \in U_{\lambda_{1}}$ and $b \in U_{\lambda_{2}}$ The set $X \backslash \overline{\left(U_{\lambda_{1}} \cup U_{\lambda_{2}}\right)}$ is countable, so it follows easily that $X$ is weakly-Lindelof. Note that this space $X$ is also almost-Lindelof.

Below we will give the construction of an example of a weakly-Lindelof space that it is not almostLindelof

PROPOSITION 3.6. A topological space $X$ is almost-Lindelöf if and only if every family $\left\{C_{\lambda}\right\}_{\lambda \in \Lambda}$ of closed subsets of $X$ such that $\bigcap_{\lambda \in \Lambda} C_{\lambda}=\emptyset$ admits a countable subfamily such that $\bigcap_{n \in \mathbb{N}}{\stackrel{\circ}{C_{n}}}_{\lambda_{n}}=\emptyset$

PROOF. If $\left\{C_{\lambda}\right\}_{\lambda \in \Lambda}$ is a family by closed subsets of $X$ such that $\cap_{\lambda \in \Lambda} C_{\lambda}=\emptyset$, then the family $\left\{X \backslash C_{\lambda}\right\}_{\lambda \in \Lambda}$ is an open cover of $X$. By hypothesis there exists a countable subfamily such that $\bigcup_{n \in \mathbf{N}} \overline{X \backslash C_{\lambda_{n}}}=X$, i e $\underset{n \in \mathbb{N}}{\cap}{\stackrel{\circ}{\lambda_{n}}}_{\lambda_{n}}=\emptyset$. Conversely, let $\left\{U_{\lambda}\right\}_{\lambda \in \Lambda}$ be an open cover of $X$ Then $\left\{X \backslash U_{\lambda}\right\}_{\lambda \in \Lambda}$ is a family by closed sets such that $\cap_{\lambda \in \Lambda}\left(X \backslash U_{\lambda}\right)=\emptyset$ By hypothesis there exists a countable subfamily such that $\bigcap_{n \in \mathbf{N}} \operatorname{int}\left(X \backslash U_{\lambda_{n}}\right)=\emptyset$, i e $X=\bigcup_{n \in \mathbb{N}} \overline{U_{\lambda_{n}}}$ This completes the proof.

PROPOSITION 3.7. Let $X$ be a topological space For the following conditions

(i) $X$ is almost-Lindelöf,

(ii) every regularly open cover $\left\{U_{\lambda}\right\}_{\lambda \in \Lambda}$ admits a countable subfamily such that $X=\cup_{n \in \mathbf{N}} \overline{U_{\lambda_{n}}}$,

(iii) every family $\left\{C_{\lambda}\right\}_{\lambda \in \Lambda}$ of regularly closed subsets of $X$ such that $\bigcap_{\lambda \in \Lambda} C_{\lambda}=\emptyset$ admits a countable subfamily such that $\bigcap_{n \in \mathbf{N}}{\stackrel{\circ}{\lambda_{n}}}_{\lambda_{n}}=\emptyset$;

we have that (i) $\Rightarrow$ (ii) $\Rightarrow$ (iii) $\Rightarrow$ (ii) and if $X$ is semiregular then (ii) $\Rightarrow$ (i)

PROOF. (i) $\Rightarrow$ (ii) is obvious by the definition. The proof of (ii) $\Leftrightarrow$ (iii) is quite similar to the proof of Proposition 36 replacing open cover with a regularly open cover of $X$ We will prove the implication (ii) $\Rightarrow$ (i) when $X$ is semiregular. Let $\left\{U_{\lambda}\right\}_{\lambda \in \Lambda}$ be an open cover of $X$. By hypothesis we can suppose that any $U_{\lambda}$ is regularly open, then there exists a countable subfamily $\left\{U_{\lambda_{n}}\right\}_{n \in \mathbb{N}}$ such that $\underset{n \in \mathbb{N}}{\cup} \overline{U_{\lambda_{t}}}=X$

This completes the proof. 
THEOREM 3.8. A weakly-Lindelof, semiregular and nearly paracompact space $X$ is almostLindelof

PROOF. Let $\left\{U_{\lambda}\right\}_{\lambda i \text { A }}$ be a cover of $X$ by regularly open sets Since $X$ is nearly paracompact, this cover admits a locally finite refinement $\left\{V_{\gamma}\right\}_{\gamma \in \Gamma^{\prime}}$ Since $X$ is weakly-Lindelof then there exists a countable subfamily such that $X=\overline{\cup_{n \in \mathbb{N}} V_{\gamma_{n}}}$ Since the family $\left\{V_{\gamma_{n}}\right\}_{n<N}$ is locally finite, then $\overline{U_{n} V_{\gamma_{n}}}=\bigcup_{n \in \mathbb{N}} \overline{V_{\gamma_{n}}}[2,11111] \quad$ Choosing, for each $n \in \mathbb{N}, \lambda_{n} \in \Lambda$ such that $V_{\imath}, \subset U$., we obtain $X=\cup_{n \in \mathbb{N}} \overline{V_{\gamma_{n}}}=\cup_{n \in \mathbb{N}} \overline{U_{\gamma_{n}}}$ By Proposition $37 X$ is almost-Lindelof

PROPOSITION 3.9 [9] An almost regular space is an almost-Lindelof space if and only if it is nearly-Lindelof

\section{CONSTRUCTION OF A WEAKLY-LINDELÖF SPACE}

LEMMA 3.10. The real line $\mathbb{R}$ can be partitioned in the union of a family, of cardinality $2^{\kappa_{0}}$, by countable dense and pairwise disjoint subsets of $\mathbb{R}$

PROOF. Let $\mathbb{Q}$ be the set of the rational numbers Consider the following equivalence relation on $\mathbb{R}$

$$
x \sim y \text { if and only if } x-y \in \mathbb{Q} .
$$

The partition of $\mathbb{R}$ so obtained is the one that we want, in fact every equivalence class is of the form $x+\mathbb{Q}$, where $x \in \mathbb{R}$, and it is a countable dense subset of $\mathbb{R}$

EXAMPLE 3.11. Let $\mathbb{R}$ be the real line and $\tau$ the usual topology on it By the previous lemma we can represent $\mathbb{R}$ as a union of continuum many countable dense and pairwise disjoint subsets of $\mathbb{R}$ We can write this partition as $\mathbb{R}=\left(\cup_{\imath \in I} S_{\imath}\right) \cup S_{0}$, where the set $I$ has cardinality $2^{\aleph_{0}} \quad$ Let $\tau_{1}$ be the topology on $\mathbb{R}$ having the base $\left\{S_{\imath}\right\}_{\imath \in I} \cup \mathbb{R}$ Let $\sigma$ be the topology generated by $\tau$ and $\tau_{1}$ and let $X=(\mathbb{R}, \sigma)$ We will show that $X$ is not almost-Lindelof Since $S_{0}$ is countable, we can write $S_{0}=\left\{x_{1}, x_{2}, \ldots, x_{n}, \ldots\right\}$ Consider the open cover of $X$

$$
X=\left(\cup_{\imath \in I} S_{\imath}\right) \cup\left(\cup_{n \in \mathbb{N}}\right] x_{n}-\frac{1}{n^{2}}, x_{n}+\frac{1}{n^{2}}[) .
$$

Suppose that $X$ is almost-Lindelöf, then there exists a countable set $\left\{i_{1}, i_{2}, \ldots, i_{n}, \ldots\right\} \subset I$ such that

$$
X=\left(\cup_{n \in \mathbb{N}} \overline{S_{\imath_{n}}}\right) \cup\left(\cup_{n \in \mathbb{N}}\left[x_{n}-\frac{1}{n^{2}}, x_{n}+\frac{1}{n^{2}}\right]\right)=\cup_{n \in \mathbb{N}}\left(S_{\imath_{n}} \cup S_{0}\right) \cup\left(\cup_{n \in \mathbb{N}}\left[x_{n}-\frac{1}{n^{2}}, x_{n}+\frac{1}{n^{2}}\right]\right) .
$$

Since the Lebesgue measure of the set $\cup_{n \in \mathbf{N}}\left[x_{n}-\frac{1}{n^{2}}, x_{n}+\frac{1}{n^{2}}\right]$ is finite, then $X \backslash\left(\cup_{n \in \mathbf{N}}\left[x_{n}-\frac{1}{n^{2}}, x_{n}+\frac{1}{n^{2}}\right]\right)$ has cardinality greater than $\aleph_{0}$ But $X \backslash\left(\cup_{n \in \mathbb{N}}\left[x_{n}-\frac{1}{n^{2}}, x_{n}+\frac{1}{n^{2}}\right]\right) \subset \underset{n \in \mathbf{N}}{\cup}\left(S_{i_{n}} \cup S_{0}\right)$ and, since the second member is countable, we obtain a contradiction. We will show now that $X$ is weakly-Lindelof Let $\left\{U_{\lambda}\right\}_{\lambda \in \Lambda}$ be an open cover of $X$ and $S_{0}=\left\{x_{0}, x_{1}, \ldots, x_{n}, \ldots\right\}$ as above. Since in the topology $\sigma$ every point of $S_{0}$ has the same fundamental system of neighborhoods as in the topology $\tau$, then for each $n \in \mathbb{N}$ there exist an open set $V_{n}$ in $\tau$ and an index $\lambda_{n} \in \Lambda$ such that $x_{n} \in V_{n} \subset U_{\lambda_{n}}$ The set $V=\bigcup_{n \in \mathbb{N}} V_{n}$ is open in $\tau$ and $S_{0} \subset V \subset \cup_{n \in \mathbb{N}} U_{\lambda_{n}}$. Let $x_{\imath} \in S_{\imath}$. For any $\tau$-open neighborhood $V_{\imath}$ of $x_{\imath}$ it is $V_{\imath} \cap S_{0}=\emptyset$ (because $S_{0}$ is dense in $(\mathbb{R}, \tau)$ ) So $V_{\imath} \cap V \neq \emptyset$, hence $S_{\imath} \cap V_{\imath} \cap V \neq \emptyset$ and this shows that $x_{\imath} \in \operatorname{cl}_{\sigma}(V)$ We obtain that $X=\operatorname{cl}_{\sigma}(V)=\operatorname{cl}_{\sigma}\left(\cup_{n \in \mathbb{N}} U_{\lambda_{n}}\right)$ and therefore $X$ is weakly-Lindelof

\section{§4. ALMOST REGULAR-LINDELÖF SPACES}

The previous example suggests some interesting remarks But before it is useful to recall the following definitions

DEFINITION 4.1 [1] An open cover $\left\{U_{\lambda}\right\}_{\lambda \in \Lambda}$ of a topological space $X$ is said to be regular if for every $\lambda \in \Lambda$ there exists a non-empty regularly closed subset $C_{\lambda}$ of $X$ such that $C_{\lambda} \subseteq U_{\lambda}$ (i e $U_{\lambda}$ is quasi regular open) and $\cup_{\lambda \in \Lambda} \stackrel{\circ}{C}_{\lambda}=X$ 
DEFINITION 4.2 [1] A topological space $X$ is said to be weakly compact if every regular cover admits a finite subfamily such that the union is dense in $X$

LEMMA 4.3. Let $X$ be the space in Example 311 Let $C$ be a regularly closed and $A$ an open set such that $C \subset A$ Then $\operatorname{int}_{\imath}(C) \subset \operatorname{int}_{7} \operatorname{cl}_{o}(A)$

PROOF. We denote with $x_{0}$ and $x_{\imath}$ the elements of $S_{0}$ and $S$, respectively We show before that if $x_{0} \in$ int $_{o}(C)$ then $x_{0} \in$ int $_{1} \mathrm{cl}_{o}(A)$ Since the fundamental system of neighborhoods of $x_{0}$ is the same whether in the topology $\sigma$ or in $\tau$, then the lemma is true Now let $x_{\imath} \in \operatorname{int}_{o}(C)$ There exists a $\tau$-open neighborhood $V_{\imath}$ of $x$, such that $V, \cap S_{\imath} \subset C$ We will show that $V_{\iota} \subset \operatorname{cl}_{\iota}(A)$ Let $x_{0} \in V$, and let $V_{11}$ be an arbitrary $\sigma$-open, and therefore $\tau$-open, neighborhood of $x_{0}$ Since $x_{0} \in V_{\imath} \cap V_{0}$, we have $V, \cap V_{0} \neq \emptyset$ and thus $S_{\iota} \cap V, \cap V_{0} \neq \emptyset$ This shows that $x_{0} \in \operatorname{cl}_{o}\left(V_{\iota} \cap S_{\imath}\right) \subset C \subset \operatorname{cl}_{l}(A) \quad$ Let $x_{\jmath} \in V_{\imath}$ Suppose that $x_{\jmath} \notin \mathrm{cl}_{o}(A)$, i e there exists a $\tau$-open neighborhood $V_{\jmath}$ of $x_{\jmath}$ such that $V_{\jmath} \cap S_{\jmath} \cap A=\emptyset$ Since $x_{\jmath} \in V_{J} \cap V_{\iota}$, then $V_{J} \cap V_{\imath} \neq \emptyset$ and therefore $V_{\jmath} \cap V_{\imath} \cap S_{0} \neq \emptyset$ Let $x_{0} \in V_{\jmath} \cap V_{1}$ We have seen above that $x_{0} \in C \subset A \subset \mathrm{cl}_{o}(A)$, since $A$ is $\sigma$-open hence there exists a $\sigma$-open, and therefore $\tau$-open neighborhood $V_{0}$ of $x_{0}$ such that $V_{0} \subset A$ Since $V_{0} \cap V_{2} \cap V_{\jmath} \neq \emptyset$ then, by density of $S_{\jmath}$, $V_{0} \cap V_{\imath} \cap V_{\jmath} \cap S_{\jmath} \neq \emptyset$ and therefore $A \cap V_{\jmath} \cap S_{\jmath} \neq \emptyset$ that is a contradiction So it is shown that $x_{\imath} \in \mathrm{cl}_{o}(A)$ and therefore $V_{\imath} \subset \mathrm{cl}_{\sigma}(A)$ The proof is complete

PROPERTY 4.5. The space $X$ in Example 311 satisfies the following property every regular cover $\left\{U_{\lambda}\right\}_{\lambda \in \Lambda}$ of $X$ admits a countable subfamily $\left\{U_{\lambda_{n}}\right\}_{n \in \mathbb{N}}$ such that $X=\cup_{n \in \mathbb{N}} \overline{U_{\lambda_{n}}}$

PROOF. Let $\left\{U_{\lambda}\right\}_{\lambda \in \Lambda}$ be a regular cover of $X$ For any $\lambda \in \Lambda$ there exists a regularly closed $C_{\lambda} \subset U_{\lambda}$ such that $X=\bigcup_{\lambda \in \Lambda} \operatorname{int}_{\sigma}\left(C_{\lambda}\right)$ By the previous lemma we have $X=\cup_{\lambda \in \Lambda} \operatorname{int}_{\tau} \mathrm{cl}_{\sigma}\left(U_{\lambda}\right)$ and, since $X$ is Lindelof with respect to the topology $\tau$, there exists a countable subcover such that $X=\bigcup_{n \in \mathbb{N}}$ $\operatorname{int}_{\tau} \operatorname{cl}_{\sigma}\left(U_{\lambda_{*}}\right)=\cup_{n \in \mathbb{N}} \operatorname{cl}_{\sigma}\left(U_{\lambda_{n}}\right)$.

The previous property suggests us to give a new definition that generalizes the weakly-Lindelof property

DEFINITION 4.6. A topological space $X$ is called almost regular-Lindelof if every regular cover $\left\{U_{\lambda}\right\}_{\lambda \in \Lambda}$ of $X$ admits a countable subfamily such that $X=\cup_{n \in N} \overline{U_{\lambda_{n}}}$

REMARK 4.7. Obviously almost-Lindelof implies almost regular-Lindelof, but the converse in general is not true, in fact the space $X$ in Example 3.11 is almost regular-Lindelof but not almostLindelof

THEOREM 4.8. An almost regular-Lindelof and almost regular space $X$ is nearly-Lindelof

PROOF. Let $\left\{U_{\lambda}\right\}_{\lambda \in \Lambda}$ be a cover by regularly open sets of $X$ For each $x \in X$ there exists $\lambda_{\mathbf{x}} \in \Lambda$ such that $\mathrm{x} \in U_{\lambda_{x}}$ Since $X$ is almost regular, there exist two regularly open subsets $V_{\lambda_{x}}$ and $W_{\lambda_{x}}$ such that $x \in V_{\lambda_{x}} \subset \overline{V_{\lambda_{x}}} \subset W_{\lambda_{x}} \bar{W}_{\lambda_{x}} \subset U_{\lambda_{x}}\left[13\right.$, Th 22] The family $\left\{W_{\lambda_{x}}\right\}_{x \in X}$ is a regular cover of $X$ and, since $X$ is almost regular-Lindelöf, there exists a countable set of points $x_{1}, x_{2}, \ldots, x_{n}, \ldots$ of $X$ such that $X=\underset{x \in \mathbb{N}}{\cup} \overline{W_{\lambda_{x}}}$ So $X=\cup_{n \in \mathbb{N}} U_{\lambda_{x n}}$ and therefore $X$ is nearly-Lindelof

The previous theorem implies the following

COROLLARY 4.9. Let $X$ be an almost regular space Then $X$ is almost regular-Lindelof if and only if it is nearly-Lindelof

We give now a characterization of almost regular-Lindelof spaces

THEOREM 4.10. A topological space $X$ is almost regular-Lindelof if and only if for every family $\left\{C_{\lambda}\right\}_{\lambda \in \Lambda}$ of closed subsets of $X$, such that for each $\lambda \in \Lambda$ there exists an open set $A_{\lambda} \supset C_{\lambda}$ with $\bigcap_{\lambda \in \Lambda} \overline{A_{\lambda}}=\emptyset$, there exists a countable subfamily such that $\bigcap_{n \in \mathbb{N}}{\stackrel{\circ}{\lambda_{n}}}_{\lambda_{n}}=\emptyset$

PROOF. Let $\left\{C_{\lambda}\right\}_{\lambda \in \Lambda}$ be a family of closed sets of $X$ such that for each $\lambda \in \Lambda$ there exists an open set $A_{\lambda} \supset C_{\lambda}$ with $\underset{\lambda \in \Lambda}{\cap} \overline{A_{\lambda}}=\emptyset \quad$ It follows that $X=X \backslash\left(\underset{\lambda \in \Lambda}{\cap} \overline{A_{\lambda}}\right)=\underset{\lambda \in \Lambda}{\cup}\left(X \backslash \overline{A_{\lambda}}\right) \quad$ But, since $C_{\lambda} \subset A_{\lambda} \subset \stackrel{\circ}{A_{\lambda}} \subset \overline{A_{\lambda}}$, then $X \backslash \overline{A_{\lambda}} \subset X \backslash \stackrel{\circ}{A_{\lambda}} \subset X \backslash C_{\lambda}$, and therefore $X=\cup_{\lambda \in \Lambda}\left(X \backslash C_{\lambda}\right) \quad$ The family 
$\left\{X \backslash C_{\lambda}\right\}_{\lambda \in \Lambda}$ is a regular cover of $X$, since $X$ is almost regular-Lindelof, then there exists a countable subfamily such that

$$
X=\underset{n \in \mathbb{N}}{\cup}\left(\overline{X \backslash C_{\lambda_{n}}}\right)=\underset{n \in \mathbb{N}}{\cup}\left(X \backslash{\stackrel{\circ}{\lambda_{\lambda_{n}}}}\right)=X \backslash\left({ }_{n \in \mathbb{N}}{\stackrel{\circ}{\lambda_{\lambda_{n}}}}\right)
$$

and therefore $\cap_{n \in \mathbb{N}}{\stackrel{\circ}{\lambda_{n}}}_{n^{\prime}}=\emptyset \quad$ Conversely, let $\left\{U_{\lambda}\right\}_{\lambda \cdot \Lambda}$ be a regular cover of $X \quad$ For each $\lambda \in \Lambda$ there exists a regularly closed $C_{\lambda}$ of $X$ such that $\stackrel{\circ}{C}_{\lambda} \subset C_{\lambda} \subset U_{\lambda}$ and $\cup_{\lambda \in \Lambda} \stackrel{\circ}{C}_{\lambda}=X$ The family $\left\{X \backslash U_{\lambda}\right\}_{\lambda \in .}$ of closed sets is such that, for each $\lambda \in \Lambda$, there exists the open set $X \backslash C_{\lambda} \supset X \backslash U_{\lambda}$ and such that

$$
\underset{\lambda \in \Lambda}{\cap} \overline{X \backslash C_{\lambda}}=\underset{\lambda \in \Lambda}{\cap}\left(X \backslash \stackrel{\circ}{C}_{\lambda}\right)=X \backslash\left(\underset{\lambda \in \Lambda}{\cup}{\stackrel{\circ}{C_{\lambda}}}\right)=X \backslash X=\emptyset .
$$

By hypothesis, there exists a countable set of indices $\left\{\lambda_{n}\right\}_{n \in \mathbb{N}}$ such that $\bigcap_{n \in \mathbb{N}} \operatorname{int}\left(X \backslash U_{\lambda_{n}}\right)=\emptyset$, i e $\bigcap_{n \in \mathbb{N}}\left(X \backslash \bar{U}_{\lambda_{n}}\right)=\emptyset$ So $X \backslash\left(\underset{n \in \mathbb{N}}{\cup} \overline{U_{\lambda_{n}}}\right)=\emptyset$ and therefore $X=\bigcup_{n \in \mathbb{N}} \overline{U_{\lambda_{n}}}$ This completes the proof

\section{ALMOST REGULAR-LINDELÖF SUBSPACES AND SUBSETS}

A subset $S$ of a space $X$ is said to be almost regular-Lindelof if $S$ is almost regular-Lindelof as a subspace of $X$

DEFINITION 4.11. A subset $S$ of a space $X$ is said to be almost regular-Lindelof relative to $X$ if for each family $\left\{U_{\lambda}\right\}_{\lambda \in \Lambda}$ of open sets of $X$ satisfying the condition

$$
S \subset \cup_{\lambda \in \Lambda} U_{\lambda} \text {, and }
$$

(*) for each $\lambda \in \Lambda$, there exists a nonempty regularly closed set $C_{\lambda}$ of $X$ such

$$
\text { that } C_{\lambda} \subset U_{\lambda} \text { and } S \subset \underset{\lambda \in \Lambda}{\cup} \stackrel{\circ}{\lambda}_{\lambda} \text {, }
$$

there exists a countable subset $\left\{\lambda_{n}\right\}_{n \in \mathbb{N}} \subset A$ such that $S \subset \underset{n \in \mathbb{N}}{\cup} \overline{U_{\lambda_{n}}}$

THEOREM 4.12. If $S$ is an almost regular-Lindelöf subspace of a space $X$, then $S$ is almost regular-Lindelof relative to $X$

PROOF. Let $\left\{U_{\lambda}\right\}_{\lambda \in \Lambda}$ be a cover of $S$ satisfying the condition (*) For each $\lambda \in \Lambda$, we have that $\stackrel{\circ}{C}_{\lambda} \cap S$ and $U_{\lambda} \cap S$ are open sets in $S$, and $C_{\lambda} \cap S$ is closed in $S$ The family $\left\{U_{\lambda} \cap S\right\}_{\lambda \in \Lambda}$ is an open cover of $S$ We will show that it is a regular cover of the subspace $S$ For each $\lambda \in \Lambda$, we have that $\mathrm{cl}_{S}\left(\stackrel{\circ}{C}_{\lambda} \cap S\right) \subset C_{\lambda} \cap S \subset U_{\lambda} \cap S$, where $\operatorname{cl}_{S}\left(\stackrel{\circ}{C}_{\lambda} \cap S\right)$ is regularly closed in $S$ Moreover, we have $S=\underset{\lambda \in \Lambda}{\cap}\left(\stackrel{\circ}{C}_{\lambda} \cap S\right)$ and $\stackrel{\circ}{C}_{\lambda} \cap S \subset \operatorname{int}_{S} \mathrm{cl}_{S}\left(\stackrel{\circ}{C}_{\lambda} \cap S\right)$, thus $S=\cup_{\lambda \in \Lambda} \operatorname{int}_{S} \operatorname{cl}_{S}\left(\stackrel{\circ}{C}_{\lambda} \cap S\right)$. Since $S$ is an almost regular-Lindelof subspace of $X$, there exists a countable subcover such that $S=\cup_{n \in \mathbf{N}} \operatorname{cl}_{S}\left(U_{\lambda_{n}} \cap S\right)$ Since for each $n \in \mathbb{N} \operatorname{cl}_{S}\left(U_{\lambda_{n}} \cap S\right) \subset \overline{U_{\lambda_{n}}}$, we obtain that $S \subset \underset{n \in \mathbb{N}}{\cup} \overline{U_{\lambda_{n}}}$. This shows that $S$ is almost regular-Lindelof relative to $X$

THEOREM 4.13. If every regularly closed subset of a space $X$ is almost regular-Lindelof relative to $X$, then $X$ is almost regular-Lindelof

PROOF. Let $\left\{U_{\lambda}\right\}_{\lambda \in \Lambda}$ be a regular cover of $X$. For each $\lambda \in \Lambda$, there exists a nonempty regularly closed set $C_{\lambda}$ of $X$ such that $C_{\lambda} \subset U_{\lambda}$ and $X=\cup_{\lambda \in \Lambda}{\stackrel{\circ}{C_{\lambda}}}_{\lambda} \quad$ Fix an arbitrary $\lambda_{0} \in \Lambda$ and let $\Lambda^{*}=\Lambda \backslash\left\{\lambda_{0}\right\}$ Put $K=X \backslash \stackrel{\circ}{C}_{\lambda_{0}}$, then $K$ is regularly closed in $X$ and $K \subset \cup_{\lambda \in \Lambda^{*}}{\stackrel{\circ}{C_{\lambda}}}^{\text {. Therefore }}\left\{U_{\lambda}\right\}_{\lambda \in \Lambda^{*}}$ is a cover of $K$ by open sets of $X$ satisfying the condition (*) of Definition 411 and hence there exists a countable subset $\left\{\lambda_{n}\right\}_{n \in \mathbf{N}} \subset \Lambda^{*}$ such that $K \subset \underset{n \in \mathbb{N}^{*}}{\cup} \overline{U_{\lambda_{n}}}$ So we have

$$
X=K \cup{\stackrel{\circ}{C_{\lambda_{0}}}}=K \cup \overline{U_{\lambda_{0}}}=\left(\underset{n \in \mathbb{N}^{*}}{\cup} \overline{U_{\lambda_{n}}}\right) \cup \overline{U_{\lambda_{0}}}=\underset{n \in \mathbb{N}}{\cup \overline{U_{\lambda_{n}}}} .
$$

This shows that $X$ is almost regular-Lindelof

COROLLARY 4.14. If every proper regularly closed subset of a space $X$ is almost regularLindelof, then $X$ is almost regular-Lindelof.

THEOREM 4.15. Let $X$ be an almost regular-Lindelof space If $A$ is a proper clopen subset of $X$, then $A$ is almost regular-Lindelof relative to $X$ 
PROOF. Let $\left\{U_{\lambda}\right\}_{\lambda \cdot A}$ be a cover of $A$ by open sets of $X$ satisfying the condition (*) of Definition 411 The family $\left\{U_{\lambda}\right\}_{\lambda \cdot 1} \cup(X \backslash A)$ is a regular cover of $X$ Since $X$ is almost regular-Lindelof, there exists a countable subfamily such that

$$
X=\left(\underset{n=}{\cup \overline{U_{\lambda_{1}}}}\right) \cup(\overline{X \backslash A})=\left(\underset{n \in \bar{U}_{\lambda_{*}}}{\cup}\right) \cup(X \backslash A)
$$

Therefore we have $A \subset \underset{n, \overline{U_{\lambda .}}}{\cup}$ This completes the proof

We conclude this paper introducing the following two definitions

DEFINITION 4.16. A space $X$ is called weakly regular-Lindelof if every regular cover $\left\{U_{\lambda}\right\}_{\lambda, \Lambda}$ of $X$ admits a countable subfamily such that $X=\overline{\cup_{n \in \mathbb{N}} U_{\lambda_{n}}}$

DEFINITION 4.17. A space $X$ is called nearly regular-Lindelof if every regular cover $\left\{U_{\lambda}\right\}_{\lambda \in \Lambda}$ of $X$ admits a countable subfamily such that $X=\underset{n \in \mathbb{N}}{\cup} \frac{0}{U_{\lambda_{n}}}$

Obviously we have the following implications

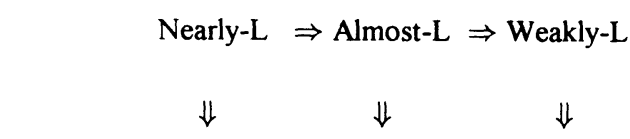

Nearly regular- $\mathrm{L} \Rightarrow$ Almost regular- $\mathrm{L} \Rightarrow$ Weakly regular- $\mathrm{L}$

We leave open the study of these two new generalizations of Lindelöf property and the relative implications

ACKNOWLEDGEMENT. This research was supported by a grant from C.N.R (G N S.A.G.A) and MU R S T through "Fondi $40 \%$ "

\section{REFERENCES}

[1] CAMMAROTO, F and LO FARO, G, Spazi weakly compact, Riv. Mat. Univ. Parma (4) 7 (1981), 383-395

[2] ENGELKING, R., General Topology, PWN, Warszawa, 1976

[3] FROLIK, Z, Generalizations of compact and Lindelöf spaces, Czechoslovak Math. J. 9 (84) (1959), 172-217 (Russian)

[4] COMFORT, W.W , HINDMAN, N. and NEGREPONTIS, S., F-spaces and their products with Pspaces, Pacific J. Math. 28 (1969), 489-502

[5] HAGER, A W, Projections of zerosets (and the fine uniformity on a product), Trans. Amer. Math. Soc. 140 (1969), 87-95

[6] ULMER, M, Products of weakly-§-compact spaces, Trans. Am. Math. Soc., 170 (1972).

[7] WOODS, G., Characterizations of some $C^{*}$-embedded subspaces of $\beta^{\mathrm{N}}$, Pacıfic J. of Math., 65, 2 (1976)

[8] BELL, M., GINSBURG, J. and WOODS, G., Cardinal inequalities for topological spaces involving the weak Lindelöf number, Pacıfic J. Math. 79 (1978), 37-45.

[9] BALASUBRAMANIAN, G, On some generalizations of compact spaces, Glasnik Mat., 17 (37) (1982), 367-380.

[10] WILLARD, S and DISSANAYAKE, U.N.B., The almost Lindelöf degree, Canad. Math. Bull., 27 (4) (1984).

[11] PAREEK, C.M, Some generalizations of Lindelöf spaces and hereditarily Lindelof spaces, $Q \& A$ in General Topology, 2 (1984)

[12] CAMMAROTO, F, Almost-homeomorphisms and almost-topological properties, Rev. Colombiana de Matemaicas, Vol. XX (1986), 51-71

[13] SINGAL, M.K and ARYA, S.P, On almost regular spaces, Glasnık Mat. Ser. III, 4 (24) (1969), 89-99

[14] SINGAL, M K. and MATUR, A, On nearly compact spaces, Boll. Un. Mat. Ital. (4), 2 (1969), 702-710

[15] SINGAL, M K and ARYA, S.P., On nearly paracompact spaces, Math. Vesnik, 6 (21) (1969), 316 


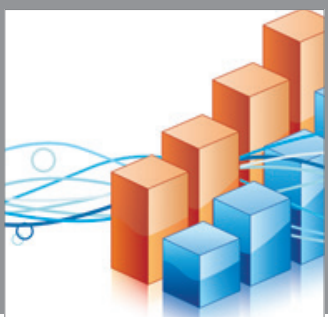

Advances in

Operations Research

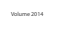

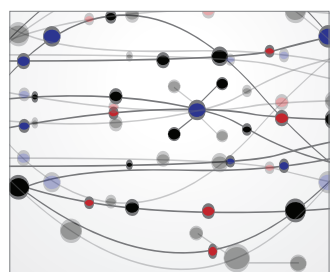

\section{The Scientific} World Journal
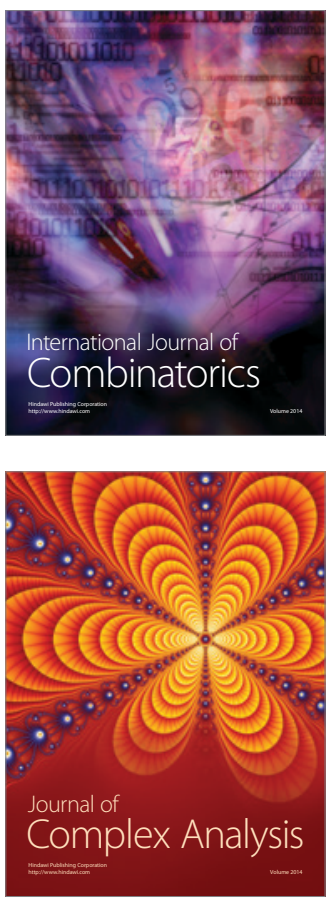

International Journal of

Mathematics and

Mathematical

Sciences
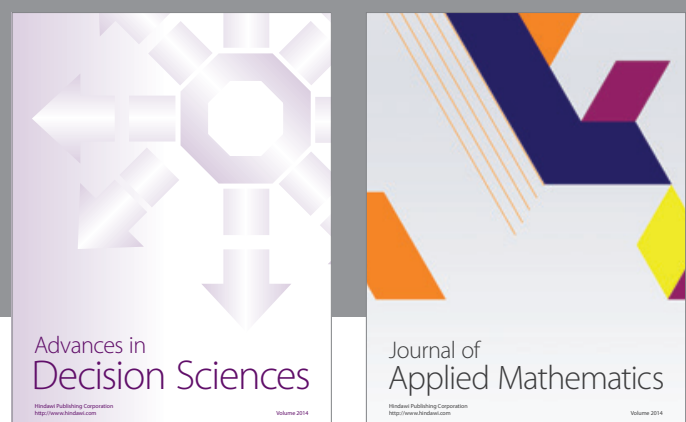

Journal of

Applied Mathematics
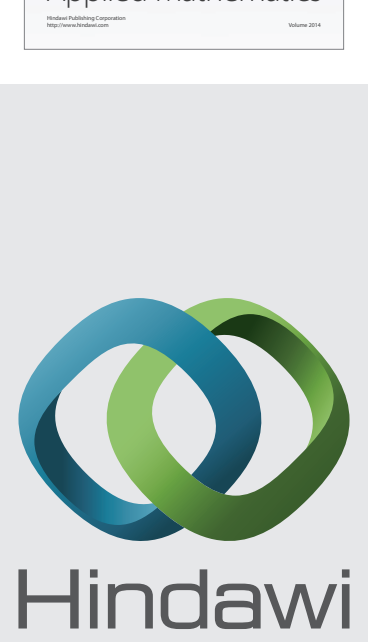

Submit your manuscripts at http://www.hindawi.com
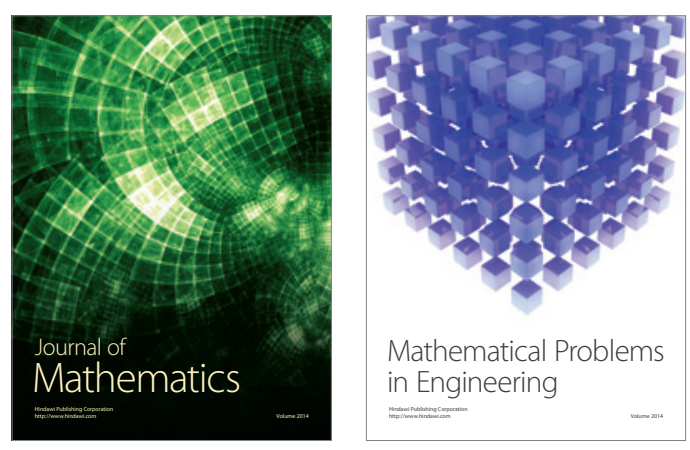

Mathematical Problems in Engineering
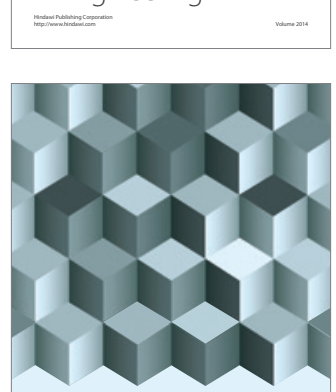

Journal of

Function Spaces
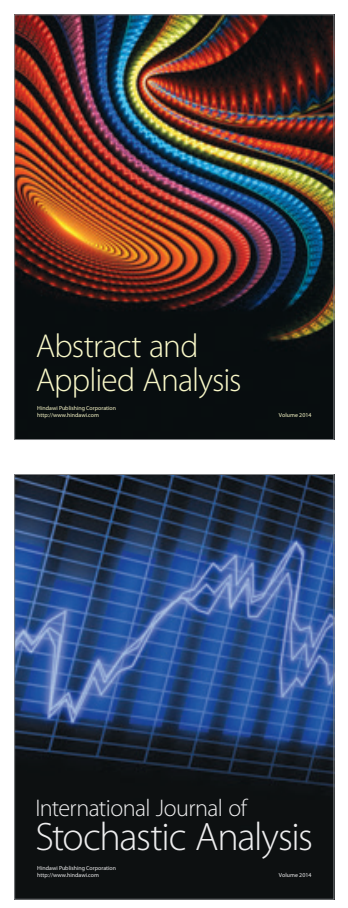

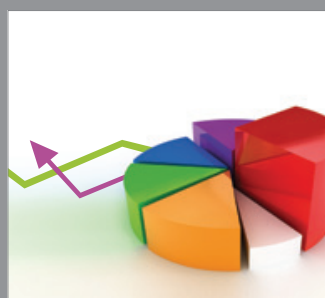

ournal of

Probability and Statistics

Promensencen
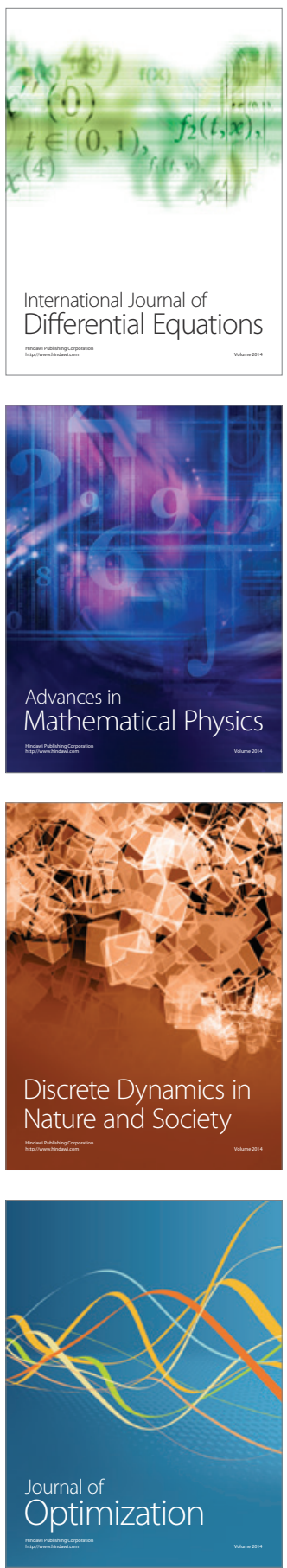